\title{
CARRIERS SPECTRA OF FUNCTIONALIZED SEMICONDUCTING NANOWIRES AND CONFORMATIONAL TRANSITION IN MOLECULES
}

\author{
V.A. LYKAKH, ${ }^{1}$ E.S. SYRKIN ${ }^{2}$ \\ ${ }^{1}$ National Technical University 'Kharkiv Polytechnic Institute' \\ (21, Frunze Str., Kharkiv 61002, Ukraine; e-mail: lykah@ilt. kharkov. ua) \\ ${ }^{2}$ B. Verkin Institute for Low Temperature Physics and Engineering, \\ Nat. Acad. of Sci. of Ukraine \\ (47, Lenin Ave., Kharkiv 61103, Ukraine; e-mail: syrkin@ilt. kharkov. ua)
}

PACS 68.65.La, 67.70. + n,

03.65.-w

(C) 2012

The tuning of the spectrum of semiconducting nanowires as a result of the functionalization by a layer of molecules with a conformational transition is investigated. The situation where the electric charge carrier induces the conformational transition with a change of the orientation of the intrinsic electric dipole moments of molecules is expected. The spectrum of a carrier and the parameters of the arising quantum well are determined by the derived selfconsistent system of transcendent equations. The system includes the Schrödinger equation for a charge carrier, nonlinear equations for the intrinsic electric-dipole moments, the material equations de4scribing the interaction of an extra carrier in the nanowire and molecular electric dipoles. In a semiconductor nanowire, the hole and electron spectra are symmetric. It is shown that the layer of adsorbed molecules breaks this symmetry when the molecular dipoles create the conditions for a localization of carriers of only one kind, which depends on the charge sign and the orientation of dipoles. The functionalized nanowires can be used as a semiconductor rectifier.

\section{Introduction}

The creation of new types of mesoscopic objects and the prospects of their application in nanoelectronics stimulate a certain interest in studies of the fundamental properties of such objects. The optical properties and the conductance of a nanoobject are determined by the set of quantum energy levels of its charge carriers [1]. The effect of the energy-level structure on the conductance has been observed for metal nanowires [2] and nanotubes [3]. A quantum nanowire can be a conventional intrinsic semiconductor or a carbon nanotube, where the mean free path of the charge carriers exceeds $10 \mu \mathrm{m}$ [4]. It is an important factor for enabling the quantization along the nanowire axis [5].

The physical properties of a nanowire are changed considerably by a medium that is in contact with it. For example, the nanowire conductance is extremely sensitive to adsorbed layers of $\mathrm{NH}_{3}$ molecules [6] and more complex molecules forming Langmuir-Blodgett films [7]. The potential for creating the chips based on nanotubes and DNA [8] or surface self-organizing layered organic structures [9] has been discussed.

Functionalization is a new powerful method for tuning the quantum energy levels of nanowires and a set of their physical properties [10]. Novel high-sensitive biosensors and electronic and optoelectronic devices including nanotubes and nanowires are created [11]. The state of molecules plays an important role. A strong conductance variation has been found in conformationally constrained molecular tunnel junctions [12]. An optically switchable device due to the conformational transition in functionalizated carbon nanotubes is created [13].

In this paper, taking the conformational transition in a molecular layer into account, we calculate the contribution to a shift of the charge-carrier energy levels that depends on the polarization of molecules. In the case of a long nanowire surrounded by a thin molecular layer, the problem of longitudinal quantization is reduced to solving the spectral problem for a Schrödinger equation. The calculation of the well parameter and the energy of the given quantum level of a charge carrier, expressed in terms of the parameter of interaction of the carrier with molecules, is reduced to solving a set of two transcendental equations. The analysis indicates that the localization of the charge-carrier motion along a nanowire is possible.

\section{Model of the System}

The time-independent Schrödinger equation for an extra carrier in an intrinsic-semiconductor nanowire can be written as $[1,14]$

$-\frac{\hbar^{2}}{2 m_{\mathrm{ef}}} \Delta \psi+U(\mathbf{r}) \psi=W \psi$. 
Here, $\psi \equiv \psi(\mathbf{r})$ is the wave function, $m_{\mathrm{ef}}$ is the charge carrier effective mass, $\Delta$ is the Laplace operator, $W$ is the total energy, $U(\mathbf{r})$ is the potential energy, and $\mathbf{r}(x, y, z)$ is the radius vector of the particle. A deep potential well can be approximated by a well of infinite depth: $U(\mathbf{r}) \equiv U_{0}(\mathbf{r})$, where $U_{0}(\mathbf{r})=0$ inside the nanowire, and $U_{0}(\mathbf{r})=\infty$ outside the nanowire. If the interaction with the surrounding medium $U_{\text {int }}(\mathbf{r})$ is taken into account, the potential is given by

$U(\mathbf{r})=U_{0}(\mathbf{r})+U_{\text {int }}^{e}(\mathbf{r})$.

This interaction can be important if the molecules possessing an intrinsic dipole moment $\mathbf{d}$ are arranged at the surface of the nanowire. Summing up the contributions from all the dipoles, we obtain the interaction potential energy $U_{\text {int }}^{e}=e \phi$ at a point $\mathbf{r}_{0}$ within the functionalizing layer:

$U_{\text {int }}^{e}\left(\mathbf{r}_{0}\right)=e \int d \mathbf{r}^{\prime} n\left(\mathbf{r}^{\prime}\right) \mathbf{d}\left(\mathbf{r}^{\prime}\right) \frac{\mathbf{r}_{0}-\mathbf{r}^{\prime}}{\varepsilon\left|\mathbf{r}_{0}-\mathbf{r}^{\prime}\right|^{3}}$.

Here, $n\left(\mathbf{r}^{\prime}\right)$ is the volume number density of the molecules, $e$ is the carrier charge, $\mathbf{d}\left(\mathbf{r}^{\prime}\right)$ is the mean value of a dipole moment at the point $\mathbf{r}^{\prime}$, and $\varepsilon$ is the relative permittivity of the medium. In turn, the strength of the electric field $\mathbf{E}\left(\mathbf{r}^{\prime}\right)$ created by a charge carrier determines the potential energy of the interaction between this carrier and an individual molecular dipole:

$U_{\text {int }}^{d}\left(\mathbf{r}^{\prime}\right)=-\mathbf{d}\left(\mathbf{r}^{\prime}\right) \int d \mathbf{r}_{0} \frac{e\left|\psi\left(\mathbf{r}_{0}\right)\right|^{2}\left(\mathbf{r}^{\prime}-\mathbf{r}_{0}\right)}{\varepsilon\left|\mathbf{r}^{\prime}-\mathbf{r}_{0}\right|^{3}}$.

System (1)-(4) is to be completed with the material equations

$n\left(\mathbf{r}^{\prime}\right)=n\left(\mathbf{E}\left(\mathbf{r}^{\prime}\right)\right) ; \quad \mathbf{d}\left(\mathbf{r}^{\prime}\right)=\mathbf{d}\left(\mathbf{E}\left(\mathbf{r}^{\prime}\right)\right)$.

\subsection{The interaction simplification}

The interaction described by (3) and (4) is nonlocal and depends substantially on the configuration of the system. The Schrödinger equation (1) with potentials (2)-(4) can be transformed into a nonlinear integro-differential equation. Such equations can be solved only by using approximation methods [14]. It is reasonable to assume that the radii of the nanowire, $r$, and the functionalizing layer, $R$, are much smaller than the nanowire length $2 l$, i.e., $r<R \ll 2 l$; this condition is satisfied in experiments (see the photo in [7]). Let us consider a system with cylindrical geometry. Here, the separation of the coordinate variables is possible under the assumption made: $\psi(\mathbf{r})=\psi(x) \psi_{\perp}(y, z)$ and $W=W_{x}+W_{\perp}$ (here, $x$ is the coordinate along the wire axis). An enhancement of the carrier tunneling into the region occupied by functionalizing molecules may result in a modification of $\psi_{\perp}(y, z)$ and $W_{\perp}$. We also assume that any variations in $\psi(x)$ occur on a length scale on the order of $l$.

The effect of the charge-carrier field is the strongest, if the molecules possess an intrinsic electric-dipole moment $\mathbf{d}$, and the molecular system is soft. We assume that the molecular system is rigid in the direction over the nanowire surface. The electric dipole moment in these molecules exists due to the presence of atomic groups that break the charge symmetry $[9,15]$. The ferroelectric ordering is possible [15], especially, if the moment is oriented by the nanowire as a substrate.

In the long-nanowire approximation, the integral contributions can be reduced to those of a local nature [16]. Let us express integral (3) in cylindrical coordinates. In the absence of charge carriers or in the case of a rigid molecular system, the density and the dipole moment along a radius in $(3)-(5)$ are constants $\left(n\left(\mathbf{r}^{\prime}\right)=n_{0}\right)$ and $d=d_{0}$. So they can be kept out of the integration sign. The integration with respect to $x$ (the coordinate along the nanowire axis) is reduced to the integration within infinite limits, even for $x_{0}-x^{\prime} \geq 3 R$. Using [18], we write the average potential energy of the interaction of a charge carrier with the dipole subsystem as

$U_{\text {int }}^{e 0}=-\frac{n_{s} e d_{0}}{\varepsilon_{0}}$,

where the thin layer (1-2 molecules) density is $n_{s}$. It is the surface density of the molecules, and $\varepsilon=1$. We obtain an approximate analytical expression for the potential energy of the interaction between a charge carrier and an individual molecular dipole. Instead of carrying out a spatial integration in (4), we calculate the fluxes [16] and make it unnecessary to specify the shape of the radial distribution. In other words, we replace the local value of the radial component of the field strength with a value calculated for an infinitely long wire taking the local value of the wave function:

$U_{\text {int }}^{d}(x)=-\mathbf{E d}=-\frac{\tau d}{2 \pi \varepsilon_{0} r^{\prime}} ;$

$\tau(x)=e|\psi(x)|^{2} F_{\perp} ; \quad F_{\perp}=\int|\psi(y, z)|^{2} d y d z$.

Here, $\tau(x)$ is the local linear charge density.

\subsection{Conformational transition (CT)}

The conformational configurations mean that a molecule has different space configurations of atomic groups. The 


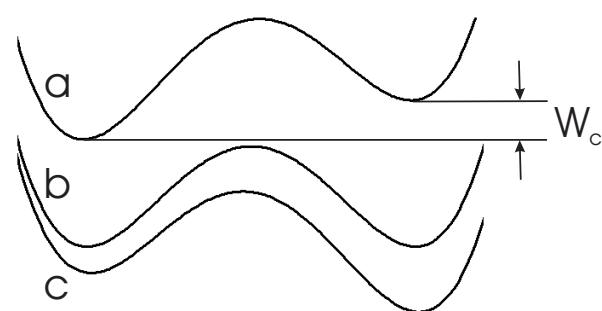

Fig. 1. Potential of a functional group with electric dipole moment in an organic molecule with a conformational transition according to (9). a) initial potential, $W_{c}$ is the energy difference between the conformational configurations of the molecule; $b$ ) the conformation potential in the critical external electric field that gives the same energies to the conformational configurations; $c$ ) the conformation potential in the external electric field which changes the conformational configuration

conformational transition changes the space configuration of the molecule. The conformational configurations may have the same values of energy as in the molecules of $\mathrm{NH}_{3}$ and trimetylenesulfide [19] or different ones as in cis- and trans-states. The conformational configurations have different energies in an asymmetric molecule in general. A number of examples of the conformational transition in vitamins B6 (pyridoxine), D3, and others can be found in [20].

Let us denote the following variables: the energy difference between the conformational configurations of an asymmetric molecule $W_{c}$, the electric dipole moments of a functionalizing molecule in the initial more stable conformation $\mathbf{d}_{0}$ and in the unstable conformation $\mathbf{d}_{1}$. Then, at CT, the electric dipole moment changing is

$\Delta \mathbf{d}=\mathbf{d}_{1}-\mathbf{d}_{0}$.

This is possible if the external electric field intensity exceeds the critical value $E_{c}$, which satisfies the relation

$-\mathbf{E}_{c} \Delta \mathbf{d}+W_{c}=0 ; \quad E_{c}=\frac{W_{c}}{\Delta d_{r}}$.

Here, $d_{r}$ is the radial projection of the electric dipole moment. The conformational transition is possible only for one mutual orientation of the vectors $\mathbf{E}$ and $\Delta \mathbf{d}$, as is shown in Fig. 1. Another mutual orientation of the vectors increases the stability of the initial conformation.

The initial molecular conformation changes a carrier potential according to relation (6) in comparison with that for a pure nanowire. After the conformational transition in functionalizing molecules, the carrier decreases its energy additionally by the value

$\Delta U \equiv \Delta U_{\text {int }}^{e}=-\frac{n_{s} e \Delta d_{r}}{\varepsilon_{0}}$.
The substitution of relation (9) into (7) can lead to the critical values of linear charge density $\tau_{c}$ and wave function density $\left|\psi_{c}\right|^{2}$ in a nanowire:

$\tau_{c}=\frac{2 \pi W_{c} \varepsilon_{0} r^{\prime}}{\Delta d_{r}} ; \quad\left|\psi_{c}\right|^{2}=\frac{\tau_{c}}{e F_{\perp}}=\frac{2 \pi W_{c} \varepsilon_{0} r^{\prime}}{F_{\perp} e \Delta d_{r}}$.

A carrier with the opposite sign of charge does not create the conformational transition. We consider independent conformational transitions in different molecules as the order-disorder phase transition in the external electric field of a carrier.

\subsection{Schrödinger equation and conformational transition}

In view of relations (6), (7), and (10), Eq. (1) can be rewritten to describe the one-dimensional motion of charge carriers in a nanowire as follows:

$-\frac{\hbar^{2}}{2 m_{\mathrm{ef}}} \frac{\partial^{2} \psi(x)}{\partial x^{2}}+U(x) \psi(x)=W_{x} \psi(x)$.

Without the conformational transition, the potential reads

$U(x)=\left\{\begin{aligned} \infty ; & |x|>l \\ 0 ; & |x| \leq l\end{aligned}\right.$

where the zero level is chosen for the energy $U_{0}+U_{\text {int }}^{e 0}$. For this potential, the problem is reduced to the wellknown quantization in a rectangle box of infinite depth $[14,17]$. After the conformational transition, we describe the appropriate carrier as an electron in the potential well of infinite depth with width $2 l$ and a symmetrically included potential well of finite depth $\Delta U(10)$ with width $2 a(a<l)$. Then the carrier potential along the nanowire is

$U(x)=\left\{\begin{array}{cr}\infty ; & |x|>l \\ 0 ; & a \leq|x| \leq l \\ -\Delta U ; & \end{array}\right.$

Here, the zero energy level is chosen as in (13), where $a$ is a half of the conformational transition region (domain) length along the nanowire. We consider a symmetric (see the discussion below) and enough deep quantum well for the final configuration of the conformational transition domain. So the basic quantum level of the carrier is inside the conformational domain well (lower than the well height).

According to [17], let us introduce the wave numbers $k_{0}$ corresponding to the conformational domain well 
depth, $k$ corresponding to the basic energy level height, and $\kappa$ corresponding to the basic energy level depth:

$\Delta U=\frac{\hbar^{2}}{2 m_{\mathrm{ef}}} k_{0}^{2} ; \quad W_{x}=\frac{\hbar^{2}}{2 m_{\mathrm{ef}}} k^{2} ; \quad \kappa^{2}=k_{0}^{2}-k^{2}$.

The wave function of the basic state is symmetric (even) and exponential outside the conformational domain well. So, according to [17], it can be written in the form

$\psi(x)= \begin{cases}A \cos k x ; & 0 \leq|x| \leq a \\ A \cos k a \frac{\sinh \kappa(l-x)}{\sinh \kappa(l-a)} ; & a \leq|x| \leq l .\end{cases}$

Here, the continuity condition at the point $x=a$ and the boundary condition $\psi(l)=0$ are used for the wave function. For the first derivation, the continuity condition at the point $x=a$ gives the equation

$\tan k a=\frac{\kappa}{k} \quad \frac{1}{\tanh \kappa(l-a)}$.

The wave function amplitude $A$ can be found from the normalization condition:

$\frac{1}{A^{2}}=a+\frac{1}{k} \sin k a \cos k a+$

$+\frac{\cos ^{2} k a}{\kappa}\left[\frac{1}{\tanh \kappa(l-a)}-\frac{\kappa(l-a)}{\operatorname{sh} \kappa(l-a)}\right]$.

\section{Self-Consistent System of Equations}

Let us write the following self-consistent system of equations:

$$
\left\{\begin{array}{l}
\kappa^{2}=k_{0}^{2}-k^{2} ; \\
\tan k a=\frac{\kappa}{k} \frac{1}{\tanh \kappa(l-a)} ; \\
\frac{1}{A^{2}}=a+\frac{1}{\kappa} \tanh \kappa(l-a)+ \\
+\frac{\cos ^{2} k a}{\kappa}\left[\frac{2}{\operatorname{sh} 2 \kappa(l-a)}-\frac{\kappa(l-a)}{\operatorname{sh}^{2} \kappa(l-a)}\right] \\
A \cos k a=\psi_{c} .
\end{array}\right.
$$

The system defines the unknown values of conformational domain quantum well size $a$, the energy $k^{2}$ of the basic quantum level, the energy depth $\kappa^{2}$ of the basic quantum level, and the amplitude $A$ of the electron wave function. The material equations give two parameters only: the depth of the conformational domain well $k_{0}^{2}$ and the critical value (amplitude) of the carrier wave function.
The first equation of system (19) determines the wave number $\kappa$ through another wave numbers (15). The second equation of the system is the the continuity condition at the point $x=a$ for the first derivation (17). The third equation of the system is the normalization condition (18) rewritten in a more convenient form for the investigation of limit cases. Thus, in the limit case $l \rightarrow \infty$, the normalization condition takes the form $1 / A^{2}=a+1 / \kappa$ for a quantum well of finite depth [17]. In the limit case $l \rightarrow a$, the normalization condition takes the form $1 / A^{2}=a$ for a quantum well of infinite depth. The fourth equation reflects the following fact: the wave function on the boundary of the conformational domain takes the critical value $\psi(a)=\psi_{c}$. The last equation (condition) directly defines the self-consistency of all sizes and energies of the problem.

The first and last equations of system (19) allow us to easily exclude the variables $A$ and $\kappa$. The rest of the system, the second and third equations, can be transformed to the following self-consistent form:

$$
\left\{\begin{array}{l}
D \tan D=\sqrt{C^{2}-D^{2}} \frac{1}{\tanh \left[\sqrt{C^{2}-D^{2}}\left(\frac{L}{C}-1\right)\right]} \\
1+\frac{1}{D \tan D}+\frac{1}{1+\tan ^{2} D}\left\{\frac{D \tan D}{C^{2}-D^{2}}-\right. \\
\left.-\frac{1}{D \tan D}-\left(\frac{L}{C}-1\right)\left(\frac{D^{2} \tan ^{2} D}{C^{2}-D^{2}}-1\right)-\frac{1}{\Phi C}\right\}=0 .
\end{array}\right.
$$

The simplification is achieved by introducing the dimensionless variables

$$
C=k_{0} a ; \quad D=k a
$$

and the dimensionless parameters

$$
L=k_{0} l ; \quad \Phi=\frac{\left|\psi_{c}\right|^{2}}{k_{0}} .
$$

The physical sense of the variables and the parameters can be clarified if they are presented in the form

$C=k_{0} a=2 \pi \frac{a}{\lambda_{0}} ; \quad D=2 \pi \frac{a}{\lambda}$.

The variable $C$ is the half-length $a$ of the conformational domain normalized to the wavelength of a carrier with the energy equal to the depth of the conformational domain well $\Delta U$. The variable $D$ is the half-length of the conformational domain normalized to the wavelength of a carrier with the basic level energy inside the conformational domain well $W$. The parameter $L$ is the halflength $l$ of the nanowire normalized on the wavelength 


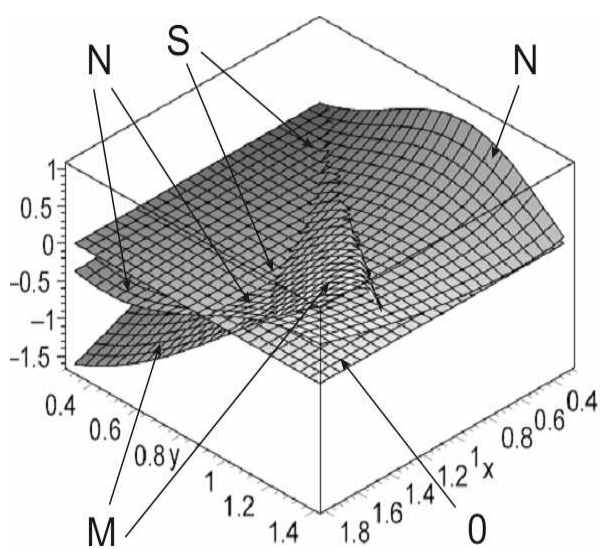

Fig. 2. A graphical representation of the system of equations (26). The dimensionless parameters are $L=2 ; \Phi=0.2$. The surfaces $z=N(C, D), z=M(C, D)$ and $z=0$ are marked as $N, M, 0$. The axis $C \equiv x, D \equiv y$. Letter $S$ marks the solution points

of a carrier with energy equal to the depth of the conformational domain well. Therefore, the variable $C$ directly gives the half-length $a$ of the conformational domain. The found half-length $a$ allows us to find $k$ and $W$ from another variable $D$. The sense of the parameter $\Phi$ is the critical probability density $\left|\psi_{c}\right|^{2}$ multiplied by the wavelength $\lambda_{0} / 2 \pi$, i.e., it is the critical probability on the interval $\lambda_{0} / 2 \pi$.

With regard for the inequalities $a \leq l$ and $k \leq k_{0}$ and the physical sense of $\Phi$, we obtain the following inequalities between the variables and parameters of the problem:

$D \leq C \leq L ; \quad 0<\Phi<1$.

In this investigation, we do not take the limit cases where the inequalities became equalities.

\section{Solution of the System of Equations}

The system of equations (20) is nonlinear and transcendental with two unknown variables. The first equation is a generalization of the one-variable equation for a finitedepth quantum well in [17] (there, $\tanh (x)$ is absent, parameter $C$ is fixed, and $k a=D$ is unknown). In [17], the equation is solved by the graphic method on a plane. We note that the numerical methods are very unstable with infinite and discontinuous functions such as $\tan (x)$ or $1 /\left(x-x_{0}\right)$, and the minimum smooth interval has to be pointed out previously.

Here, we use the graphic method. However, it must operate with for two unknown variables in the 3D space. The basic idea is to avoid periodic singularities by multiplication by $\sin D, \cos D$. Let us introduce smooth func- tions depending on two variables that correspond to Eqs. (20):

$$
\left\{\begin{array}{l}
M(C, D)=D \sin D \tanh \left[\sqrt{C^{2}-D^{2}}\left(\frac{L}{C}-1\right)\right]- \\
-\sqrt{C^{2}-D^{2}} \cos D ; \\
N(C, D)=\sin D\left[1+\frac{\sin D \cos D}{D}+\right. \\
\left.+\left(\frac{L}{C}-1-\frac{1}{\Phi C}\right) \cos ^{2} D\right]\left(C^{2}-D^{2}\right)+ \\
+D \sin ^{2} D\left[\cos D-\left(\frac{L}{C}-1\right) D \sin D\right] .
\end{array}\right.
$$

The solution of system (20) corresponds to the point of triple intersection of graphs $0, M(C, D), N(C, D)$ or a pair crossing of the intersection curves on the $C, D$ plane:

$\left\{\begin{array}{l}M(C, D)=0 \\ N(C, D)=0\end{array}\right.$

We do calculations for the following values of dimensionless parameters: $L=2 ; \Phi=0.2$. We use the standard graphic program. The direct construction of these three surfaces is not suitable. The reason is very different vertical scales. Some simplification can be due to a guide idea [17]: for the basic quantum level, the variable $D$ changes in the range $[0 ; \pi / 2]$. The ranges for the $M(C, D)$ and $N(C, D)$ functions differ by one order as minimum. Since we multiple a less value, this procedure does not change the solution points in (26) (see Fig. 2).

The next qualitative result can be obtained from the graphic analysis of the $M(C, D)$ and $N(C, D)$ functions on the $(C, D)$ plane. The surfaces can have a joint point on the surface $z=0$ on the line in Fig. 2 only. In the considered region, this line can be described with high precision as a straight line:

$D(C)=D_{0}+k\left(C-C_{0}\right) ; \quad k:=\frac{D_{1}-D_{0}}{C_{1}-C_{0}}$,

where the coordinates of the points, $C_{0} \simeq 0.74 ; D_{0} \simeq$ $0.7 ; C_{1} \simeq 1.04 ; D_{1} \simeq 0.91$, can be found from the largescale graphs. The further substitution of relation (27) into Eqs. (26) and (20) reduces the problem to that with one unknown variable:

$\left\{\begin{array}{l}M(C)=0 ; \\ N(C)=0\end{array}\right.$

Graphically, this means finding of the intersection of surfaces by the vertical plane (27). This intersection is shown at Fig. 3, $a$ in a wide range. Standard 2D 
graphic programs give a more precise presentation in comparison with $3 \mathrm{D}$ packages. On the large scale, the curve crossing is shown in Fig. 3,b. The fitting of the intersecting vertical plane $(27)$ can be executed by moving the point $D_{1}=0.9064$ which is near the crossing point. The final value of $D_{1}$ and the corresponding solutions $C_{c}$ and $D_{c}$ are obtained at the limit of the graphic resolution. They are shown in the first row of Table. The direct substitution of the values of $C_{c}$ and $D_{c}$ in (25) gives $M=1.379 \times 10^{-8}$ and $N=9.74 \times 10^{-9}$.

If we know the values of $C$ and $D$, we can find the relative values of the physical parameters:

$\frac{a}{l}=\frac{C_{c}}{L} ; \quad \frac{k}{k_{0}}=\frac{D_{c}}{C_{c}} ; \quad \frac{W}{W_{0}}=\frac{k^{2}}{k_{0}^{2}}$

Here, $a / l, k / k_{0}$, and $W / W_{0}$ are the relative conformational domain size (width of the arising quantum well), momentum, and the basic energy level, respectively. The results of calculations are shown in Table.

For other values of the dimensionless parameters, $L=10 ; \Phi=0.2$, the fitting of the intersecting plane give three solutions. Two of them are shown in the second and third rows of Table, respectively. The direct substitution of functions (25) gives $M=$ $2.658 \times 10^{-7}, N=2.635 \times 10^{-8}$ for the first and $M=-5.38 \times 10^{-9}, N=1.807 \times 10^{-5}$ for the second solutions.

It is obvious that the second solution is unstable in comparison with the first one, because of $W_{2}>W_{1}$. The third formal solution coincides with the special point of the functions $M(C, D)$ and $N(C, D)$ and is very unstable.

At small $L$ (enough shallow well), the system of equations has the only solution for the base quantum state. At large $L$ (enough deep well), the solution for the base quantum state become ambiguous. Therefore, on the line $L=2 \div 10 ; \Phi=0.2$ in the parameters' plane, a bifurcation of system (25) arises.

Initial parameters $L, \Phi$ and the results of the graphic solution $D_{c}, C_{c}$ of the self-consistent system of equations. The conformational domain relative parameters are calculated according to (29)

\begin{tabular}{c|c|c|c|c|c|c}
\hline$L$ & $\Phi$ & $C_{c}$ & $D_{c}$ & $a / l$ & $k / k_{0}$ & $W / W_{0}$ \\
\hline 2 & 0.2 & 1.0589 & 0.9193 & 0.5294 & 0.8682 & 0.7538 \\
10 & 0.2 & 1.1005 & 0.7813 & 0.1100 & 0.7100 & 0.5041 \\
10 & 0.2 & 0.2001 & 0.1966 & 0.0200 & 0.9826 & 0.9655 \\
\hline
\end{tabular}
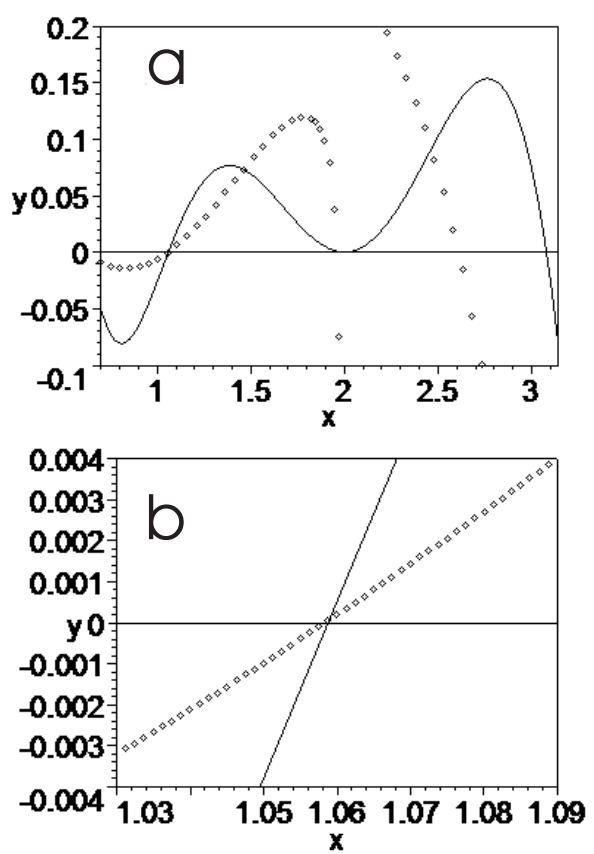

Fig. 3. Graphic solution of system (28). The axis $C \equiv x$. The horizontal straight line means $y=0$, the line curve is $y=N(C)$, and the box is the curve $y=M(C)$. (a) wide ranges of $x, y ;(b)$ the large-scale view of the curve crossing

\section{Discussion and Conclusions}

The electric dipole of a molecule and its change $\Delta d$ can be estimated as

$\Delta d \sim d \sim\left|e r_{0}\right| \simeq 6.4 \times 10^{-29} \sim 10^{-28} \mathrm{C} \cdot \mathrm{m}$

where $r_{0} \simeq 0.4 \mathrm{~nm}$ is the size of the dipole which is approximately equal to interatomic distance. The carrier energy decreasing in a functionalized nanowire after the conformational transition can be estimated according to (10) as

$\Delta U=\left|-\frac{n_{s} e \Delta d}{\varepsilon_{0}}\right| \sim\left(10^{+1}-10^{-1}\right) \mathrm{eV}$,

where the surface concentration can be estimated as $n_{s} \simeq S^{-1}$, where $S \sim\left(10^{2}-10^{4}\right) \times 10^{-20} \mathrm{~m}^{2}$ is the surface occupied by one molecule. In the experimental work [13], the surface concentration of molecules was determined as one per 100 elementary cells of the nanowire surface, and $\Delta U \simeq 1.5 \mathrm{eV}$. We take this value for further estimations.

The linear density of charge

$\tau_{c} \sim e / 2 l \sim 10^{-13} \mathrm{C} / \mathrm{m}$ 
Here, $2 l \sim 1 \mu \mathrm{m}$ is a nanowire length. The last estimation and relations (11) allow us to evaluate

$W_{c}=\frac{\tau_{c} \Delta d_{r}}{2 \pi \varepsilon_{0} r^{\prime}} \sim 10^{-3} \mathrm{eV}$.

If a nanowire is shorter $\left(2 l \sim\left(10^{-7}-10^{-8}\right) \mathrm{m}\right)$, then the critical conformational energy is higher: $W_{c} \sim\left(10^{-2}\right.$ $\left.10^{-1}\right) \mathrm{eV} \sim(100-1000) \mathrm{K}$. This means that the shorter the nanowire, the higher energy difference between the conformation configurations should be overcome by a carrier electric field.

The longitudinal quantization creates quantum levels, which have energy distance by several orders less than that for the transverse quantization. Therefore, a carrier can tunnel into the nanowire on a level with high number (high number of nodes of the wave function $\psi(x)$ ). If conditions (9) or (11) are satisfied in several space intervals, the conformational transition may go and create several additional quantum wells. Since the conformation transition in different molecules goes independently, this scenario develops by a chaotic way. Namely, the conformational transition in different wells goes by independent jumps, and the wave function along the nanowire fluctuates.

A more probable scenario is the following: first of all, a carrier goes into the ground quantum state, and then the conformational transition is realized in one domain. This scenario develops if the electron-phonon relaxation processes (characteristic time $\tau_{\mathrm{e}-\mathrm{ph}}$ ) for the carrier energy are faster than the conformational transition relaxation (characteristic time $\tau_{\mathrm{CT}}$ ):

$\tau_{\mathrm{e}-\mathrm{ph}}<\tau_{\mathrm{CT}}$.

Both these scenarios are possible if only the wave functions of a high energy level $\psi_{n}(x)$ and the ground state $\psi_{1}(x)$ exceed the critical value (11):

$\psi_{n}(x) \geq \psi_{c} ; \quad \psi_{1}(x) \geq \psi_{c}$.

The wave function of the ground state has only one maximum. Therefore, the conformational transition develops more or less chaotically from the central point with maximum electric field to the region with decreasing wave function. The conformational transition range decreases, when the conformational transition domain border reaches the points $\psi_{1}(x)=\psi_{c}$. As a result, the conformational transition domain is formed closely to the symmetric position along a nanowire as in (14).

Above, we considered a nanowire rather than a quantum dot (QD). We need to add the electrostatic energy $e^{2} / 2 C$ to the previously found energy levels. Here, $C$ is the electric capacity. The nanowire reveals its quantum levels by means of optic spectra or tunneling in contacts. At a long tunneling time of one kind of carrier, the system has enough time to transit to the state with conformational domain. This domain can be considered as a trap for the corresponding carrier. In this case, the behaviors of different carriers differ, and the system must show the rectifier property. The system shows the asymmetric current-voltage characteristic and can be used as a rectifier.

Thus, in the present work, the energy spectrum of uncompensated charge carriers in a functionalized nanowire is investigated. The interaction of a charge carrier with conformation degree of freedom in a functionalizing molecular system is considered. The physical mechanism responsible for this interaction is pointed out: the interaction of molecular dipole moments with the electric field of a charge carrier.

The self-consistent system of equations is derived for the well width and the spectrum parameters. The system including four nonlinear transcendent equations is reduced to two equations and is solved by the graphic method. With this purpose, the 3D plots are analyzed qualitatively, and the intersections are found by excluding one variable and reducing the problem to $2 \mathrm{D}$ one.

Thus, it is shown that if the well that arises at the conformational transition is enough deep, then the selfconsistent system of equations has an ambiguous solution. Nevertheless, there is the physical criterion of selection of the stable solution, namely, by the energy minimum of the system.

The charge-carrier energy spectrum depends on the rigidity of a functionalizing molecular system. At the conformational transition, the rigidity depends on the mutual orientation of the electric field strength of an uncompensated charge carrier and the electric dipole moments of molecules. The conformational transition in the electric field and a modification of the energy spectrum are possible for one sign of the carrier charge. The carrier with another sign of charge feels a homogeneous change of the potential along the nanowire only. Thus, a functionalized nanowire can be used as a semiconductor rectifier. The processes considered should be taken into account in the design of chips based on nanowires and layered organic surface structures $[8,9]$.

1. D.K. Ferry and S.M. Goodnick, Transport in Nanostructures (Cambridge Univ. Press, Cambridge, 1997). 
2. A.I. Yanson, I.K. Yanson, and J.M. van Ruitenbeek, Phys. Rev. Lett. 87, 216805 (2001).

3. D. Orlikowski, H. Mehrez, J. Taylor, H. Guo, J. Wang, and C. Roland, Phys. Rev. B 63, 155412 (2001).

4. P. Poncharal, C. Berger, Yan Yi et al., J. Phys. Chem. B 106, 12104 (2002).

5. C. Dekker, Phys. Today 52, 22 (1999).

6. H. Dai, Phys. World 13(6), 43 (2000).

7. N.P. Armitage, J.-C.P. Gabriel, and G. Gruner, J. Appl. Phys. 95, 3228 (2004).

8. E. Buzaneva, A. Gorchynskyy, G. Popova et al., Frontiers of Multifunctional Nanosystems, edited by E. Buzaneva and P. Scharff (Kluwer, Dordrecht, 2002), p. 191.

9. O. Neilands, Molecular Low Dimensional and Nanostructured Materials for Advanced Applications, edited by A. Graja et al.) (Kluwer, Dordrecht, 2002), p. 181.

10. S. Daniel, T.P. Rao, K.S. Rao et al., Sens. and Actuat. B 122, 672 (2007).

11. C.M. Lieber and Zhong Lin Wang, MRS Bull. 32, 99 (2007).

12. C.B. George, M.A. Ratner, and J.B. Lambert, J. Phys. Chem. A 113, 3876 (2009).

13. E.D. Canto, K. Flavin, M. Natali, T. Perova, and S. Giordani, Carbon 48, 2815 (2010).

14. L.D. Landau and E.M. Lifshitz, Quantum Mechanics. Non-Relativistic Theory (Pergamon Press, New York, 1980).

15. R. Blinc and B. Zeks, Soft Modes in Ferroelectrics and Antiferroelectrics (North-Holland, Amsterdam, 1974).

16. V.A. Lykakh and E.S. Syrkin, Semicond. Sci. Technol. 39, 679 (2005).
17. S. Flügge, Practical Quantum Mechanics (Springer, Berlin, 1971).

18. H.B. Dwight, Tables of Integrals and Other Mathematical Data (Macmillan, New York, 1961).

19. W.H. Flygare, Molecular Structure and Dynamics (Prentice-Hall, Englewood Cliffs, NJ, 1978).

20. D.E. Metzler, Encyclopedia of Physical Science and Technology [c]. Biochemistry (Academic Press, New York, 2001), p. 509 .

Received 08.10.11

\section{СПЕКТРИ НОСІЇВ В НАПІВПРОВІДНИКОВИХ НАНОДРОТАХ І КОНФОРМАЦІЙНИЙ ПЕРЕХІД У МОЛЕКУЛАХ}

B.О. Ликах, Є.С. Сиркін

Р е з ю м е

Досліджено перебудову спектра полупровідникових нанодротів в результаті функціоналізації шаром молекул з конформаційним переходом. Носій електричного заряду викликає конформаційний перехід зі зміною напрямку власних електричних дипольних моментів молекул. Спектр носія і параметри квантової ями, що виникає, визначаються з отриманої самоузгодженої системи трансцендентних рівнянь. Система включає рівняння Шредінгера для носія заряду, нелінійні рівняння для власних електричних дипольних моментів, матеріальні рівняння для взаємодії додаткового заряду в нанодроті і електричних диполів молекул. У напівпровідниковому нанодроті діркові й електронні спектри є симетричними. Показано, що шар адсорбованих молекул порушує цю симетрію, диполі молекул створюють умови для локалізації тільки одного виду носіїв в залежності від знака заряду та орієнтації диполів. Функціоналізовані нанодроти можуть використовуватися як напівпровідниковий випрямляч. 